\title{
Users' perceptions and preferences towards maxillary removable orthodontic retainers: a crossover randomized clinical trial
}

\section{Diego Coelho LORENZONI ${ }^{(a)}$ José Fernando Castanha HENRIQUES(a) \\ Letícia Korb da SILVA(b) \\ Arthur César de Medeiros ALVES(a)}

Giédre BERRETIN-FELIX(b)

Guilherme JANSON(a) iD

(a) Universidade de São Paulo - USP, Bauru Dental School, Department of Orthodontics, Bauru, SP, Brazil.

(b) Universidade de São Paulo - USP, Bauru Dental School, Department of SpeechLanguage Pathology, Bauru, SP, Brazil.

Declaration of Interests: The authors certify that they have no commercial or associative interest that represents a conflict of interest in connection with the manuscript.

Corresponding Author:

Diego Coelho Lorenzoni

E-mail:dclorenzoni@yahoo.com.br

https://doi.org/10.1590/1807-3107bor-2019.vol33.0078

Submitted: August 28, 2018

Accepted for publication: June 13, 2019

Last revision: June 27, 2019
Abstract: The aim of this study was to assess, correlate, and compare users' perceptions and preference related to maxillary removable retainers. Volunteers were recruited to use four retainer types: conventional wrap-around (CWA), wrap-around with an anterior opening (OWA), "U" wrap-around (UWA), and clear thermoplastic retainer $(\mathrm{CT})$. The main outcomes were the volunteers' perceptions, evaluated with a $100-\mathrm{mm}$ visual analogue scale, and their preferred retainer. The retainers were used for 21 days each (washout intervals of 7 days). Nineteen volunteers ( $27 \pm 4.53$ years) were randomly divided into four groups that used the four retainers, but with a different sequence. Perceptions were evaluated immediately after the use of each retainer and the preference at the end of the research. Repeated measures ANOVA and Friedman tests with post-hoc Tukey's test (intergroup comparisons), and Pearson and Spearman analyses (correlations between perceptions) were applied. The WA retainers did not significantly differ among themselves. The CT was rated significantly worse in speech $(p \leq 0.001)$, discomfort $(p<0.001)$, and occlusal interference $(p<0.001)$, and did not significantly differ from the others in esthetics. Users preferred significant more the WA retainers in comparison with the CT retainers. The occlusal interference caused by the CT was positively correlated to other perceptions, such as changes in speech and discomfort. WA retainers presented similar preference and perceptions, but were significantly better than the CT. The CT occlusal coverage appeared to be the primary cause of its rejection.

Keywords: Orthodontic Retainers; Orthodontic Appliance Design; Malocclusion; Visual Analog Scale; Perception.

\section{Introduction}

Several types of orthodontic retainers have been described in the literature, but there is no scientific support for the choice of the best device in clinical practice, or significant evidence that a specific retainer design has a better function than another. ${ }^{1}$ Immediately after orthodontic treatment, a full-time use of a retainer is usually indicated. ${ }^{2,3}$ Because of the uninterrupted use and as most of the maxillary retainers are 
removable, patient compliance is essential; therefore, the appliance should be comfortable. Some of the reasons reported for not using retainers include discomfort, forgetfulness, loss of the appliance, maladjustment, speech difficulties, and bad smile esthetics, but information on this topic is scarce. ${ }^{4,5}$

Discomfort of the retainer is reported by $28 \%$ of patients as a reason not to use them., ${ }^{4,5}$ Data show that clear thermoplastic retainers (CT) are more comfortable than wrap-around retainers (WA), ${ }^{6,7}$ or that comfort does not differ between CT and Hawley retainer. ${ }^{8}$ Speech interference is an important aspect of comfort, because components of the retainer are located on the lingual surface of teeth and palate, impairing tongue movements during speech. ${ }^{9,10}$ Between 10 and $15 \%$ of patients report that speech difficulty is a reason not to use the appliance. ${ }^{4}$ Esthetics is also relevant, since 17.4 and $7.2 \%$ of patients reported to be ashamed to use Hawley and CT retainers, respectively. This difference corroborates the fact that $\mathrm{CTs}$ are more esthetic than other removable retainers. ${ }^{6,8}$ On the other hand, WA is better during chewing in comparison with the $\mathrm{CT}{ }^{6}{ }^{6}$

Until now, these investigations were based on the subjective perception of different groups of patients with different retainers, which makes it susceptible to personal variations between samples. Currently, there is no randomized clinical investigation on perception of different retainers in the same group of volunteers. Therefore, the aim of this study was to evaluate, compare, and correlate the perceptions of the same group of persons to different types of maxillary removable orthodontic retainers. The null hypothesis was that perceptions and preference are not different regarding maxillary removable orthodontic retainers.

\section{Methods}

\section{Trial design and changes after trial commencement}

This randomized crossover controlled clinical trial involved four groups of volunteers randomized with a 1:1:1:1 allocation ratio. ${ }^{11}$ No change in methods were necessary after trial commencement. The trial was registered at the Brazilian Clinical Trials Registry REBEC, ensaios clínicos.gov.br (RBR-2v3k6r).

\section{Participants, eligibility criteria, and settings}

Ethical approval was obtained from the Ethics Committee of Bauru Dental School, University of São Paulo, Brazil. Consecutive volunteers were recruited at the Institution from June 2015 to August 2016. They were post-graduate students at this University. The study was conducted in accordance with the ethical principles of the Declaration of Helsinki.

An orthodontist and a speech therapist of the research team performed the selection of volunteers. Eligibility criteria were the following: both sexes, between 20-40 years of age, native Portuguese speakers, presence of the first and second permanent molars, and acceptable Class I canine occlusal relationship (variations up to $1 / 4$ of Class II or III were accepted). Exclusion criteria were the presence of anterior or posterior crossbite, a zero or greater than $4 \mathrm{~mm}$ overjet, open bite, deep bite greater than $50 \%$, space deficiency with crowding greater than $2 \mathrm{~mm}$, and widespread anterior diastemas. Additionally, the subjects could not have been in orthodontic treatment or have had it completed for less than 12 months, have used any type of maxillary retainer in the last 12 months or any type of retainer in the mandibular arch (except the flat mandibular canine-to-canine bonded retainer). Finally, the presence of edentulous spaces, craniofacial anomalies, intellectual deficits, syndromes, neurological or psychiatric disorders, smoking, alcohol consumption, previous laryngeal surgery, altered lingual frenulum, active temporomandibular disorder, and pathological vocal alteration were also exclusion criteria.

\section{Interventions}

An orthodontist of the research team performed the interventions from August 2015 to January 2016. At the initial orthodontic exam, participants were invited to participate in the study and provided written informed consent after receiving a full explanation of the study objectives and structure. After that, alginate impressions of their maxillary arches were taken to obtain dental casts on which the retainers were made and participants were randomly allocated into four groups that used the four retainers in a different sequence. The same experienced professional manufactured all 
appliances. The wrap-around clasp was reused in all wrap-around retainers for a volunteer, switching only the acrylic part to minimize interferences in the research. The four retainers are described below and shown in Figure 1:

a. Conventional wrap-around (CWA): A vestibular arch wire of $0.9 \mathrm{~mm}$ stainless steel was constructed contouring the center of the maxillary teeth crowns until the most posterior tooth, around the distal surface at the gingival margin, reaching the palate, where the retention was made. Simple cervical loops were constructed between the canines and first premolars. The palatal acrylic had a thickness between 2.5 and $3 \mathrm{~mm}$, with the anterior limit covering the cervical third of the anterior teeth, the lateral limit covering the cervical of the posterior teeth, and the posterior limit extending to a line connecting the first molars distal surfaces.

b. Wrap-around with an anterior opening (OWA): The vestibular arch wire, the acrylic thickness, and its anterior and lateral limits were similar to the CWA. The posterior limit of the palatal acrylic extended to a line connecting the first molars mesial surfaces. The acrylic had an opening in the region of the palatine wrinkles, to guide the tongue position and function, and to facilitate speech. The dimensions of this opening are shown in Figure 1B.

c. Horseshoe-shaped or " $U$ " wrap-around (UWA): The vestibular arch wire, the acrylic thickness, and its anterior and lateral limits were similar to the CWA. There was no acrylic in the central portion of the palate and its dimensions are shown in Figure 1C. It was slightly wider in the anterior area and slightly thicker than the CWA to provide more resistance, but not exceeding $3 \mathrm{~mm}$.

d. Clear thermoplastic retainer (CT): Made of 1-mm thick clear Essix ${ }^{\circledR}$ thermoplastic material (Dentsply, York, USA), vacuum-molded to all maxillary teeth, including occlusal surfaces. The cervical limits were $2 \mathrm{~mm}$ above the gingival margins on the palatal and buccal surfaces.

Each type of maxillary retainer was used for 21 days ( \pm 1 day) by all participants of the four groups most of the time, except during meals, sleeping, and social events. Between each retainer, participants had a washout period without any appliance for 1 week. Therefore, all volunteers used the four retainers, each group in a different sequence. This characterized the crossover design of the study.

The volunteers' perceptions to several factors during the use of the different appliances were investigated using a Visual Analogue Scale (VAS). A sheet of paper containing 7 100-mm scales (one for each perception assessed) were given to participants and they were instructed to make a perpendicular line on the scale at the point corresponding to the desired score (ranging from 0 to 100) for each evaluated item. A 15-mm rule (Trident; Itapuí, São Paulo, Brazil) was used to measure the scores in millimeters, from the left end of the scale to the mark. The VAS was applied to all volunteers immediately after the end of the period using each retainer. They had no access to the
A

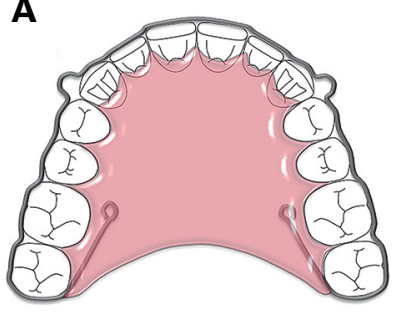

B

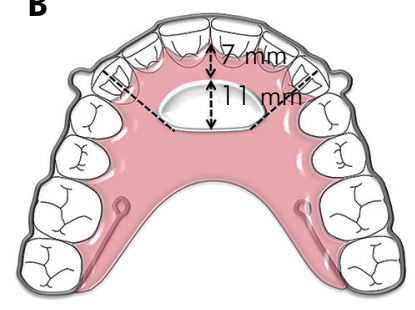

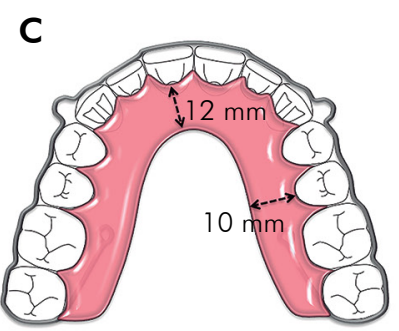

D

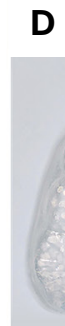

Figure 1. A. Conventional wrap-around retainer; B. Wrap-around retainer with an anterior opening. C. Horseshoe-shaped or " $U$ " wrap-around retainer. D. Clear thermoplastic retainer. 
VAS results of the previous retainer. The perceptions evaluated were:

a. Speech: Zero meant no change and 100 the maximum change in speech.

b. Swallowing: Zero meant no difficulty and 100 the maximum difficulty in swallowing.

c. Esthetics: Zero meant no impairment and 100 the maximum impairment to esthetics.

d. Salivation: Zero meant no increase in salivation and 100 the maximum increase in salivation.

e. Nausea: Zero meant no symptom and 100 the maximum nausea.

f. Discomfort: Zero meant maximum comfort and 100 the maximum level of discomfort.

g. Occlusion: Zero meant no interference and 100 the maximum level of occlusal interference.

\section{Outcomes}

Primary outcomes were the perceptions described above, registered in the $100 \mathrm{~mm}$ VAS. At the end of the research, participants were questioned about the general preference among the four retainers, giving a score from 1 to 4 , from the best to worst. There were no outcome changes after trial commencement.

\section{Sample size calculation}

Sample size calculation was based on the ability to detect an intergroup difference in discomfort perception of $20 \mathrm{~mm}$ in the VAS, with a standard deviation of $24.3 \mathrm{~mm}$ (greater standard deviation observed in a previous similar study), ${ }^{12}$ an alpha error of $5 \%$, and a test power of $80 \%$. The StatsToDo was used to calculate sample size for the analysis of variance, available on the website (https://www. statstodo.com/SSizAOV_Pgm.php). Seventeen participants were required in each group. It should be emphasized that the sample size calculation was performed for ANOVA. Because this was a crossover study, all the same volunteers participated in the four research groups.

\section{Randomization}

A simple computer-generated randomization with stratification by gender was accomplished with a 1:1:1:1 ratio for the four groups, according to the retainer sequence, using theStata ${ }^{\odot}$ software (StataCorp,
College Station, TX) to ensure equal distribution of participants in the groups. Allocation concealment was achieved with sequentially numbered and sealed envelopes containing the orthodontic retainer sequence allocation cards, which were prepared before the trial. One operator (D.C.L.) was responsible for opening the sequence of envelopes and implementing the randomization process.

\section{Blinding}

Blinding of patients and operator regarding the type of appliance was not possible; however, the outcome assessment was blinded because VAS records were unidentified during the analysis. One operator (D.C.L.) performed all the measurements on the VAS.

\section{Statistical analyses}

Normal distribution of each variable was verified using Shapiro-Wilk test. Differences in perceptions of patients concerning the four retainers were investigated with repeated measures ANOVA and post-hoc Tukey's test, for normally distributed data, or Friedman test and post-hoc Tukey's test, for data with non-normal distribution. These analyses were conducted with the SigmaPlot $12^{\odot}$ (Systat Software Inc., San Jose, USA). A statistical significance level of $5 \%$ was regarded for all tests.

Correlation between the different perceptions about each type of retainer was investigated with Pearson's correlation coefficient. These correlations were conducted with the Statistical Package for Social Sciences, version 21 (SPSS Inc., Chicago, USA).

\section{Results}

\section{Participant flow and baseline data}

Forty-seven participants were recruited from June 2016 to August 2016; 20 (42.5\%) patients were excluded because they did not meet the eligibility criteria and $6(12.7 \%)$ because they declined to participate. Twenty-one volunteers, 13 men and 8 women with a mean age of 26.7 years (standard deviation \pm 4.3 ), were included in this investigation. The sample size obtained allowed a dropout rate of $19 \%$. Figure 2 shows a flowchart with reasons of losses and exclusions before and after randomization. 


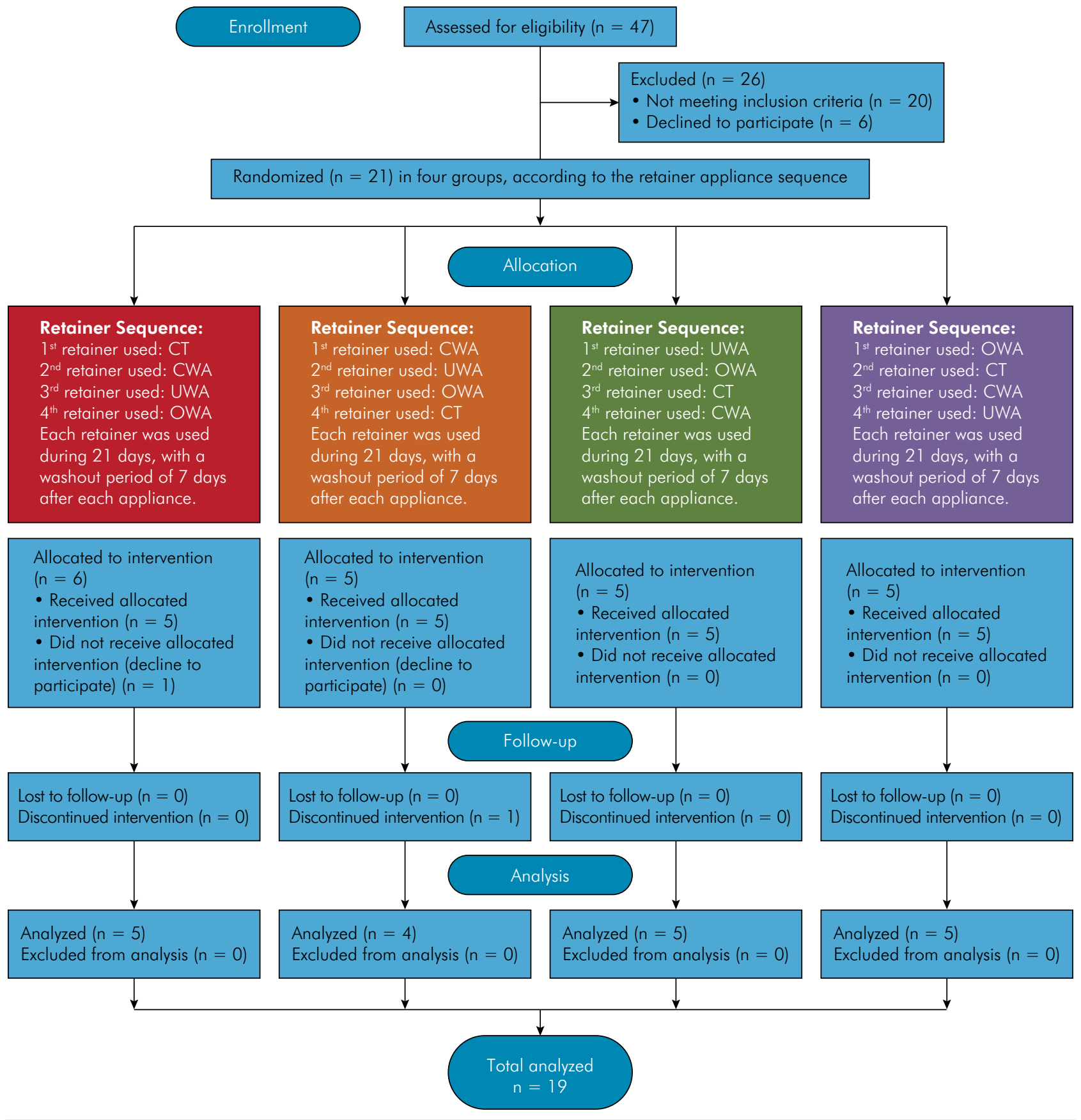

Figure 2. Flow diagram of study recruitment and interventions. CWA: Conventional Wrap-Around retainer; OWA: Wrap-Around retainer with an opening; UWA: "U" Wrap-Around retainer; CT: Clear Thermoplastic retainer.

\section{Numbers analyzed for each outcome}

Two participants (one man and one woman) dropped out (9.5\%) immediately after the randomization process because they scheduled a long trip during the survey period. Thus, the final sample had 19 participants, 12 men and 7 women, with a mean age of 27 years (standard deviation \pm 4.53 ).
Descriptive statistics of the participants' perceptions are shown in Table 1, as well as the results of statistical comparisons between retainers for each perception. Users' preference of retainers is described in Table 2. In an overall assessment, the UWA presented better scores and higher preference, but with no statistically significant difference in comparison to other WA 
designs. The UWA was preferred by 10 of 19 participants, but this was not enough to generate statistical difference from other WAs.

In general, the CT presented significantly worse perceptions, except in esthetics, which showed no significant difference compared to other WA retainers.
The CT was chosen as the worst option by 15 of the 19 volunteers, differing significantly from all the other WAs.

Correlation results can be seen in Table 3. Discomfort was positively correlated with speech changes on all retainers. Rejection to CT was positively correlated with

Table 1. Descriptive statistics of the perceptions of the four retainers.

\begin{tabular}{|c|c|c|c|c|c|}
\hline \multirow{2}{*}{ Variable } & \multirow{2}{*}{ Mean (SD) } & Mean & \multirow{2}{*}{ Median (IR) } & Median & Statistics \\
\hline & & $95 \% \mathrm{Cl}$ & & $95 \% \mathrm{Cl}$ & (p-value) \\
\hline \multicolumn{6}{|l|}{ Speech } \\
\hline CWA & $28.89(22.6)^{a}$ & $19.1-39.2$ & $26.5(38.5)$ & $10.5-40.5$ & \multirow{4}{*}{$\begin{array}{l}\text { Repeated measures } \\
\text { ANOVA }\left(p<.001^{*}\right)\end{array}$} \\
\hline OWA & $32.86(26.0)^{b}$ & $22.2-45.0$ & $25.0(46.5)$ & $12.5-40.5$ & \\
\hline UWA & $28.47(22.2)^{a}$ & $20.0-38.9$ & $22.0(31.5)$ & $14.0-31.5$ & \\
\hline CT & $63.78(25.4)$ & $52.3-74.6$ & $69.5(33.0)$ & $53.0-72.0$ & \\
\hline \multicolumn{5}{|c|}{ Swallowing } & \multirow{5}{*}{ Friedman $\left(p=.010^{*}\right)$} \\
\hline CWA & $15.36(24.8)$ & $5.3-26.8$ & $2.5(22.0)$ & $0.0-13.0$ & \\
\hline OWA & $15.78(18.9)$ & $8.1-23.9$ & $11.0(22.5)$ & $0.0-20.0$ & \\
\hline UWA & $7.05(10.1)$ & $3.0-12.1$ & $0.0(11.0)^{c}$ & $0.0-10.0$ & \\
\hline $\mathrm{CT}$ & $26.68(24.0)$ & $16.5-37.4$ & $17.5(43.5)$ & $10.5-50.0$ & \\
\hline \multicolumn{5}{|l|}{ Esthetics } & \multirow{5}{*}{ Friedman $(p=.079)$} \\
\hline CWA & $29.92(17.9)$ & $21.5-38.1$ & $22.5(37.0)$ & $16.5-49.0$ & \\
\hline OWA & $23.68(16.9)$ & $16.1-31.4$ & $23.5(28.5)$ & $14.0-32.5$ & \\
\hline UWA & $25.81(17.5)$ & 18.3-33.7 & $23.5(22)$ & $17.5-31-5$ & \\
\hline $\mathrm{CT}$ & $18.18(20.1)$ & $9.5-26.5$ & $9.5(38.5)$ & $0.0-33.0$ & \\
\hline \multicolumn{5}{|c|}{ Salivation } & \multirow{5}{*}{ Friedman $\left(p<.001^{*}\right)$} \\
\hline CWA & $21.02(22.4)$ & $11.4-31.9$ & $20.5(29.5)^{a}$ & $3.0-27.0$ & \\
\hline OWA & $24.07(25.7)$ & $13.7-35.8$ & $17.0(33.0)^{a}$ & $5.0-31.0$ & \\
\hline UWA & 10.05 (12.3) & 5.0-16.3 & $6.0(14.5)^{\mathrm{a}}$ & $0.0-11.0$ & \\
\hline $\mathrm{CT}$ & $61.73(34.6)$ & $45.5-76.4$ & $76.0(60.5)$ & $37.0-90.0$ & \\
\hline \multicolumn{5}{|c|}{ Discomfort } & \multirow{5}{*}{$\begin{array}{l}\text { Repeated measures } \\
\text { ANOVA }\left(p<.001^{*}\right)\end{array}$} \\
\hline CWA & $29.07(26.9)^{a}$ & $17.9-41.4$ & $19.5(36.0)$ & $13.0-40.0$ & \\
\hline OWA & $25.57(30.9)^{a}$ & $13.6-39.0$ & $14.5(25.0)$ & $9.5-24.5$ & \\
\hline UWA & $21.28(20.2)^{a}$ & $13.3-30.4$ & $13.0(30.5)$ & $9.0-31.5$ & \\
\hline $\mathrm{CT}$ & $67.23(25.9)$ & $55.9-78.9$ & $72.0(43.0)$ & $54.0-83.0$ & \\
\hline \multicolumn{5}{|l|}{ Nausea } & \multirow{5}{*}{ Friedman $\left(p=.002^{*}\right)$} \\
\hline CWA & $8.23(20.6)$ & $1.3-17.9$ & $0.0(4.5)$ & $0.0-2.5$ & \\
\hline OWA & $7.10(22.9)$ & $0.4-17.8$ & $0.0(3.5)$ & $0.0-2.0$ & \\
\hline UWA & $0.52(1.2)$ & $0.0-1.0$ & $0.0(0.0)^{c}$ & $0.0-0.0$ & \\
\hline $\mathrm{CT}$ & 16.71 (28.3) & $5.2-29.9$ & $8.5(13.0)$ & $0.0-12.0$ & \\
\hline \multicolumn{5}{|c|}{ Occlusion } & \multirow{5}{*}{ Friedman $\left(p<.001^{*}\right)$} \\
\hline CWA & 9.47 (18.7) & $2.6-18.4$ & $0.0(10.0)^{a}$ & $0.0-10.0$ & \\
\hline OWA & 11.57 (19.2) & $4.2-21.0$ & $0.0(20.0)^{a}$ & $0.0-20.0$ & \\
\hline UWA & $11.57(19.2)$ & $4.2-21.0$ & $0.0(20.0)^{a}$ & $0.0-20.0$ & \\
\hline $\mathrm{CT}$ & 75.78 (22.9) & $65.7-86.3$ & $80.0(50.0)$ & $60.0-100$ & \\
\hline
\end{tabular}

*Statistically significant difference. SD: STANDARD DEVIATION; IR: interquartile range; Cl: confidence interval; CWA: conventional wrap-around; OWA: wrap-around with an opening; UWA: "U" wrap-around; CT: clear thermoplastic retainer; Post-hoc Tukey test: ap $<.001$ vs. Essix; $b p=.001$ vs. Essix; $c p<.05$ vs. Essix. 
Table 2. Users' preference of retainers from the best $\left(1^{\text {st }}\right)$ to the worst $\left(4^{\text {th }}\right)$.

\begin{tabular}{|c|c|c|c|c|c|c|}
\hline \multirow{2}{*}{ Retainer } & \multicolumn{4}{|c|}{ Preference } & \multirow{2}{*}{ Total $n$} & \multirow{2}{*}{$\begin{array}{c}\text { Mean (SD) } \\
95 \% \mathrm{Cl}\end{array}$} \\
\hline & $1^{\text {st }}$ & $2^{\text {nd }}$ & $3^{\text {rd }}$ & $4^{\text {th }}$ & & \\
\hline \multirow{2}{*}{ UWA } & \multirow{2}{*}{10} & \multirow{2}{*}{5} & \multirow{2}{*}{2} & \multirow{2}{*}{2} & \multirow{2}{*}{19} & $1.78(1.03)^{* a}$ \\
\hline & & & & & & $1.36-2.26$ \\
\hline \multirow{2}{*}{ OWA } & \multirow{2}{*}{5} & \multirow{2}{*}{7} & \multirow{2}{*}{6} & \multirow{2}{*}{1} & \multirow{2}{*}{19} & $2.10(0.93)^{* a}$ \\
\hline & & & & & & $1.68-2.47$ \\
\hline \multirow{2}{*}{ CWA } & \multirow{2}{*}{3} & \multirow{2}{*}{6} & \multirow{2}{*}{9} & \multirow{2}{*}{1} & \multirow{2}{*}{19} & $2.31(0.88)^{* b}$ \\
\hline & & & & & & $1.94-2.68$ \\
\hline \multirow{2}{*}{ CT } & \multirow{2}{*}{1} & \multirow{2}{*}{1} & \multirow{2}{*}{2} & \multirow{2}{*}{15} & \multirow{2}{*}{19} & $3.63(0.83)$ \\
\hline & & & & & & $3.26-3.94$ \\
\hline
\end{tabular}

Totaln 19 - 19 : 19

SD: standard deviation; Cl: confidence interval; CWA: conventional wrap-around; OWA: wrap-around with an opening; UWA: “U” wrap-around; CT: clear thermoplastic retainer); *Repeated measures ANOVA ( $<0.001)$; Post-hoc Tukey test: ap $<.001$ vs. Essix; bp =.002 vs. Essix.

speech changes, discomfort, and occlusal interference. In addition to rejection, occlusal interference with CT showed a significant positive correlation with discomfort. In the OWA, changes in swallowing were positively correlated with salivation, discomfort, and nausea. The UWA was the favorite retainer for most participants, but its worst scores for preference were correlated to greater changes in swallowing.

\section{Harms}

A variable pressure sensation and mild pain in teeth were reported by the first participants during initial use of the CT. After this intercurrence, all $\mathrm{CT}$ retainers were redone with application of three layers of nail varnish on the teeth of the cast model. No more symptoms were reported.

\section{Discussion}

\section{Main findings in the context of the existing evidence and their interpretation}

Until now, there was no crossover study on perceptions of orthodontic retainers in which patients are their own controls. All previous studies have evaluated perceptions on different groups, which reduces the importance of the comparisons. $4,5,6,7,8,9,12,13,14,15,16,17$ The most commonly used maxillary orthodontic retainers are removable, especially the Hawley plate, the WA, and the CT, whose preferences vary according to country or region. ${ }^{2,3,16,18,19,20,21,22,23}$ The WA was selected for this study in relation to the Hawley plate, because the aim was to evaluate the influence of the different palatal acrylic designs, and the occlusal interference generated by the Hawley retaining clasps could impair this evaluation.

Table 3. Significant correlations between perceptions on different retainers.

\begin{tabular}{ll}
\hline Slgnificant correlations & \\
\hline Conventional Wrap-Around & $.668^{* .002}$ \\
Speech X Discomfort & $.529^{* .02}$ \\
Salivation X Esthetics & \\
Wrap-Around with an opening & $.471^{* .042}$ \\
Speech X Discomfort & $.490^{* .033}$ \\
Speech X Preference & $.832^{* .001}$ \\
Swallowing X Salivation & $.639^{* .003}$ \\
Swallowing X Discomfort & $.470^{* .042}$ \\
Swallowing X Nausea & $.631^{* .004}$ \\
Salivation X Discomfort & $.463^{* .046}$ \\
Salivation X Nausea & $.502 * .028$ \\
Discomfort X Nausea & \\
“U” Wrap-Around & $.585^{* .008}$ \\
Speech X Salivation & $.699^{* .001}$ \\
Speech X Discomfort & $.499 * .03$ \\
Swallowing X Preference & \\
Clear Thermoplastic Retainer & $.626^{* .004}$ \\
Discomfort X Speech & $.526^{* .021}$ \\
Discomfort X Salivation & $.889^{* .001}$ \\
Discomfort X Occlusion & $.692^{* .001}$ \\
Discomfort X Preference & $.814^{* .001}$ \\
Occlusion X Speech & $.640^{* .003}$ \\
Occlusion X Salivation & $.488^{* .034}$ \\
Occlusion X Preference & $.512^{* .025}$ \\
\hline Preference X Speech & \\
\hline Statistical significance. & \\
\hline
\end{tabular}

*Statistical significance. 
Thinner maxillary removable retainers appear to generate less interference in speech. ${ }^{13}$ Thicknesses of up to $5 \mathrm{~mm}$ have been described for the $\mathrm{WA}^{17}$ but this study used a thinner acrylic of around $2.5 \mathrm{~mm}$, balancing appliance resistance and comfort to the patient, in agreement with recent data. ${ }^{15} \mathrm{~A}$ thickness of $1 \mathrm{~mm}$ was chosen for the CT because it is used by most orthodontists. ${ }^{16}$ Moreover, a CT thinner than $1 \mathrm{~mm}$ presents a higher fracture rate and does not appear to be more comfortable. ${ }^{24}$

Three weeks of appliance use is a very short time compared to the usual time of the retention phase. However, we enrolled volunteers who were not in retention or in active orthodontic treatment to test the four different retainers with washout periods because this study design was unfeasible in patients under orthodontic treatment. To avoid participants' exhaustion and a greater dropout rate, the period of time was restricted to three weeks. Each additional week of use of each appliance would mean a month more of observation for volunteers and, in a prospective clinical study, the risk of dropout should be considered. Also, for this reason, the retainers were not used during sleep, because speech does not occur and swallowing is reduced during this time. Furthermore, all models were used in the beginning, middle, and end of the research, to minimize any possible influence. Additionally, the daily time of use was not controlled with questionnaires or chips embedded in the appliances, ${ }^{25}$ to reproduce the clinical routine, which usually does not include such protocols.

In general, the UWA received better scores but did not significantly differ from the CWA and OWA. The UWA seemed to be less uncomfortable and cause less changes in speech than the CWA. ${ }^{12}$ On the other hand, the CT received significantly worse scores than the other retainers in all perceptions, except in esthetics, a result that differs from the literature in several respects. The CT has been considered more esthetic than other retainers. ${ }^{6,8}$ In this study, the $\mathrm{CT}$ also obtained better scores for esthetics, but without significant difference compared to other appliances, similarly to a published research in which the CT and Hawley retainers were compared. ${ }^{4}$ Some explanations for this surprising fact can be given. Firstly, other perceptions could have been so negative in the CT (speech, swallowing, salivation, discomfort, nausea and occlusion) that esthetics lost its relevance. Another possibility could be related to the complaint about saliva accumulation in the plate-tooth interface and its unpleasant appearance in the anterior region. Finally, the CT used in this research was not completely clear, but translucent or slightly milky, as described by the manufacturer, a characteristic that may have negatively impacted the esthetic perception. It is noteworthy that among the many factors reported for the lack of compliance in using the retainers (forgetfulness, inconvenience in placing and removing the retainer, discomfort, loss of the retainer, speech changes), esthetics seems to have the lowest importance for patients. ${ }^{4,5}$

Another highlighted perception was the discomfort caused by retainers, a fact described by $28 \%$ of patients as a reason not to use it. ${ }^{4,5}$ The CT seemed more comfortable than the WA, ${ }^{6}$ and comfort did not differ between the CT and the Hawley plate. ${ }^{8}$ Among the WA appliances, the UWA received better scores, but these retainers did not differ significantly among themselves. On the other hand, the CT was significantly more uncomfortable than all WA. Some reasons for the great discomfort caused by the CT can be inferred from correlations with other perceptions, since its discomfort was positively correlated with speech changes, salivation, and especially with the occlusal interference caused by the occlusal coverage, which is not present in the WA.

The occlusal interference generated by the occlusal coverage appeared to be the major cause of negative perceptions and the rejection of the CT in relation to the other WAs, as it was very positively correlated to other perceptions like speech changes, salivation, and discomfort. In addition, due to the occlusal coverage of the CT, the increase in the number of occlusal contacts during retention after fix appliance removal seems to hamper vertical adjustment and the increase in contact number during retention, which usually happens with the WA or Hawley plate. ${ }^{26,27}$ Another issue is the soft characteristic of the material with which the CT is made. It is suggested that, differently from the hard occlusal splints, those made of soft material do not reduce muscle activity 
but, oppositely, seem to stimulate it in patients with nocturnal bruxism. ${ }^{28}$ For this reason, the CT should be avoided in these patients.

The CT should be extended over the entire maxillary arch to prevent extrusion of the most posterior teeth, which could lead to an anterior open bite. The need to encompass the most posterior teeth aggravates the occlusal interference, often beyond the limits of the freeway space, a fact reported by some volunteers, particularly those who presented third molars. Thus, it is suggested to extend the retainer only to the middle of the last teeth, which prevents their extrusion and minimizes posterior occlusal interference. The CT also seems to deteriorate more easily due to pressure of the teeth, especially in patients with bruxism, and incorporates fluids and pigments, which lead to color change over time. ${ }^{4}$ Unfortunately, due to the short period of use of each retainer (21 days), this fact could not be observed. At the beginning of the study, some patients reported that the CT pressed the teeth and gums in different places. This justified the retainer construction after relieving the teeth of the model, without extending to the gum.

Speech was positively correlated with discomfort in all appliances, especially in the CT, which was significantly more uncomfortable than the others. This result confirms the literature where speech changes are reported by $15 \%$ of patients as the reason for not using the retainer. ${ }^{4,5}$ Moreover, it justifies the efforts to explore and seek solutions for this problem., ${ }^{9,13,14,15,17}$

The null hypothesis was rejected because the WA retainers did not significantly differ from each other, but presented significantly better perceptions and preference compared to the CT. The occlusal interference appeared to be the major cause of negative perceptions and the rejection of the CT. Therefore, WA retainers should be given preference for the retention phase of orthodontic treatment. Further studies would be interesting to confirm these results in orthodontic patients with a longer usage time.

\section{Limitations}

This investigation presents some limitations. First, the duration of use of the retainer was short (21 days) in comparison to the usual retention period. It is known that some changes, such as in speech, tend to greatly improve after 1 month of retainer use, but may still be present after 3 months. ${ }^{9}$ Secondly, the perceptions were evaluated by the volunteers themselves, and not by specialists. Finally, the volunteers were not orthodontic patients and were not using any appliance before entering this study. On the other hand, this fact also reduces the influence of previous appliance experiences and the effect of possible treatment relapses of the results.

The use of the VAS to numerically evaluate perception could be considered a limitation in this research. However, the VAS is a reliable ${ }^{29}$ and commonly used scoring method in health research to generate parametric data from subjective notions, such as pain ${ }^{30}$ and smile attractiveness. ${ }^{31}$

\section{Generalizability}

These results are important because they reveal the perceptions of the users, who are the most important part in the retention phase with removable appliances, when compliance is essential. However, generalization of these results to orthodontic patients should be performed with caution, since the volunteers were not orthodontic patients and their perceptions may be slightly different. In addition, the retainers were used for a short period of time (21 days), and the perceptions may change with a longer time. Finally, since the volunteers were adults, these results should be carefully applied to children, whose adaptation to retainers may be different.

\section{References}

1. Littlewood SJ, Millett DT, Doubleday B, Bearn DR, Worthington HV. Retention procedures for stabilising tooth position after treatment with orthodontic braces. Cochrane Database of Syst Rev. 2016 Jan; 29(1):CD002283. https://doi.org/10.1002/14651858.CD002283.pub4

2. Valiathan M, Hughes E. Results of a survey-based study to identify common retention practices in the United States. Am J Orthod Dentofacial Orthop. 2010 Feb;137(2): 170-7. https://doi.org/10.1016/i.ajodo.2008.03.023 
3. Ab Rahman N, Low TF, Idris NS. A survey on retention practice among orthodontists in Malaysia. Korean J Orthod. 2016 Jan;46(1):36-41. https://doi.org/10.4041/kjod.2016.46.1.36

4. Pratt MC, Kluemper GT, Hartsfield JK Jr, Fardo D, Nash DA. Evaluation of retention protocols among members of the American Association of Orthodontists in the United States. Am J Orthod Dentofacial Orthop. 2011 Oct;140(4):520-6. https://doi.org/10.1016/j.ajodo.2010.10.023

5. Lin F, Sun H, Ni Z, Zheng M, Yao L. A feasible method to improve adherence of Hawley retainer in adolescent orthodontic patients: a randomized controlled trial. Patient Prefer Adherence. 2015 Oct;9:1525-30. https://doi.org/10.2147/PPA.S94111. eCollection 2015

6. Kumar AG, Bansal A. Effectiveness and acceptability of Essix and Begg retainers: a prospective study. Aust Orthod J. 2011 May;27(1):52-6.

7. Saleh M, Hajeer MY, Muessig D. Acceptability comparison between Hawley retainers and vacuum-formed retainers in orthodontic adult patients: a single-centre, randomized controlled trial. Eur J Orthod. 2017 Aug;39(4):453-61. https://doi.org/10.1093/ejo/cjx024

8. Hichens L, Rowland H, Williams A, Hollinghurst S, Ewings P, Clark S et al. (2007) Cost-effectiveness and patient satisfaction: Hawley and vacuum-formed retainers. Eur J Orthod. 2007 Aug;29(4):372-38. https://doi.org/10.1093/ejo/cjm039

9. Kulak Kayikci ME, Akan S, Ciger S, Ozkan S. Effects of Hawley retainers on consonants and formant frequencies of vowels. Angle Orthod. 2012 Jan;82(1):14-21. https://doi.org/10.2319/032911-226.1

10. Chen J, Wan J, You L. Speech and orthodontic appliances: a systematic literature review. Eur J Orthod. 2018 Jan;40(1):29-36. https://doi.org/10.1093/ejo/cjx023

11. Moher D, Hopewell S, Schulz KF, Montori V, Gotzsche PC, Devereaux PJ et al. CONSORT 2010 explanation and elaboration: updated guidelines for reporting parallel group randomized trials. Int J Surg. 2012;10(1):28-55. https://doi.org/10.1016/j.ijsu.2011.10.001

12. Choi JH, Moon CH. Discomfort caused by the circumferential comfortable retainer (CCR) as a removable maxillary retainer. Korean J Orthod. 2010 Oct; 40(5):325-33. https://doi.org/10.4041/kjod.2010.40.5.325

13. Erb DP. Speech effects of the maxillary retainer. Angle Orthod. 1967 Oct;37(4):298-303. https://doi.org/10.1043/0003-3219(1967)037<029 8:SEOTMR $>2.0 . \mathrm{CO} ; 2$

14. Feldman EW. Speech articulation problems associated with placement of orthodontic appliances. J Speech Hear Disord. 1956 Mar;21(1):34-8.

15. Haydar B, Karabulut G, Ozkan S, Aksoy AU, Ciger S. Effects of retainers on the articulation of speech. Am J Orthod Dentofacial Orthop. 1996 Nov;110(5):535-40. https://doi.org/10.1016/S0889-5406(96)70062-8

16. Meade MJ, Millett D. Retention protocols and use of vacuum-formed retainers among specialist orthodontists. J Orthod. 2013 Dec;40(4):318-25. https://doi.org/10.1179/1465313313Y.0000000066

17. Stratton CS, Burkland GA. The effect of maxillary retainers on the clarity of speech. J Clin Orthod. 1993 Jun;27(6):338-40.

18. Assumpção WA, Ota GKB, Ferreira RI, Cotrim-Ferreira FA. Orthodontic retainers: analysis of prescriptions sent to laboratories. Dental Press J Orthod. 2012 Mar-Apr;17(2):36.el-6. http://dx.doi.org/10.1590/S2176-94512012000200007

19. Lai CS, Grossen JM, Renkema AM, Bronkhorst E, Fudalej PS, Katsaros C. Orthodontic retention procedures in Switzerland. Swiss Dent J. 2014;124(6):655-61.

20. Lima, V.S.A., Carvalho, F.A.R., Almeida, R.C.C. and Capelli Jr, J. Different strategies used in the retention phase of orthodontic treatment. Dental Press J Orthod. 2012 July-Aug;17(4):115-21. http://dx.doi.org/10.1590/S2176-94512012000400022

21. Renkema AM, Sips ET, Bronkhorst E, Kuijpers-Jagtman AM. A survey on orthodontic retention procedures in The Netherlands. Eur J Orthod. 2009 Aug;31(4):432-37. https://doi.org/10.1093/ejo/cjn131

22. Singh P, Grammati S, Kirschen R. Orthodontic retention patterns in the United Kingdom. J Orthod. 2009 Jun;36(2):115-21. https://doi.org/10.1179/14653120723040

23. Wong P, Freer TJ. Patients' attitudes towards compliance with retainer wear. Aust Orthod J. 2005 May;21(1):45-53.

24. Zhu Y, Lin J, Long H, Ye N, Huang R, Yang X et al. Comparison of survival time and comfort between 2 clear overlay retainers with different thicknesses: A pilot randomized controlled trial. Am J Orthod Dentofacial Orthop. 2017 Mar; 151(3):433-9. https://doi.org/10.1016/i.ajodo.2016.10.019

25. Hyun P, Preston CB, Al-Jewair TS, Park-Hyun E, Tabbaa S. Patient compliance with Hawley retainers fitted with the SMART((R)) sensor: a prospective clinical pilot study. Angle Orthod. 2015 Mar;85(2):263-9. https://doi.org/10.2319/030814-163.1

26. Hoybjerg AJ, Currier GF, Kadioglu O. Evaluation of 3 retention protocols using the American Board of Orthodontics cast and radiograph evaluation. Am J Orthod Dentofacial Orthop. 2013 Jul;144(1):16-22. https://doi.org/10.1016/i.ajodo.2013.02.022

27. Sauget E, Covell Jr DA, Boero RP, Lieber WS. Comparison of occlusal contacts with use of Hawley and clear overlay retainers. Angle Orthod. 1997 Jun;67(3):223-30.

28. Okeson JP. The effects of hard and soft occlusal splints on nocturnal bruxism. J Am Dent Assoc. 1987 Jun;114(6):788-91. https://doi.org/10.14219/jada.archive.1987.0165

29. Howells DJ, Shaw WC. The validity and reliability of ratings of dental and facial attractiveness for epidemiologic use. Am J Orthod. 1985 Nov;88(5): 402-8. https://doi.org/10.1016/0002-9416(85)90067-3

30. Michelotti A, Farella M, Gallo LM, Veltri A, Palla S, Martina R. Effect of occlusal interference on habitual activity of human masseter. J Dent Res, 2005 Jul;84(7): 644-8. https://doi.org/10.1177/154405910508400712

31. Paiva TT, Machado RM, Motta AT, Mattos CT. Influence of canine vertical position on smile esthetic perceptions by orthodontists and laypersons. Am J Orthod Dentofacial Orthop. 2018 Mar; 153(3): 371-6. https://doi.org/10.1016/j.ajodo.2018.04.011 